symptomatic intracranial hemorrhage, or ambulate independently at discharge. They were more likely to die or be discharged to hospice.

Conclusions Though use of EVT in GWTG-Stroke for in-hospital stroke remains low, it more than doubled in the past decade. Compared with community onset stroke, these patients have longer intervals to CT and arterial puncture, with associated worse functional outcomes. While there may be important differences in baseline patient characteristics between the groups, efforts must still be made to shorten time to reperfusion for in-hospital strokes.

Disclosures F. Akbik: None. H. Xu: None. Y. Xian: None. S. Shah: None. E. Smith: None. D. Bhatt: None. R. Matsouaka: None. G. Fonarow: None. L. Schwamm: None.

\section{E-056 THE 'CLOT' SUMMIT - FIRST 5 YEARS OF A FOCUSED INCUBATOR PLATFORM FOR GUIDING STROKE RESEARCH}

${ }^{1} \mathrm{~A}$ Rai ${ }^{*},{ }^{2} \mathrm{~J}$ Fiehler, ${ }^{3} \mathrm{R}$ McCarthy, ${ }^{4} \mathrm{~A}$ Siddiqui, ${ }^{5} \mathrm{D}$ Liebeskind, ${ }^{6} \mathrm{~W}$ Hacke. ${ }^{1}$ INTERVENTIONAL NEURORADIOLOGY, WEST VIRGINIA UNIVERSITY, Morgantown, WW; ${ }^{2}$ NEURORADIOLOGY, University Medical Center Hamburg-Eppendorf, Hamburg, GERMANY; ${ }^{3}$ Scientific Affairs, Cerenovus, Miami, FL; ${ }^{4}$ Neurosurgery, University of Buffalo, Buffalo, NY; ${ }^{5}$ Neurology, University of California, Los Angeles, Los Angeles, CA; ${ }^{6}$ Neurology, University of Heidelberg, Heidelberg, GERMANY

\subsection{6/neurintsurg-2020-SNIS.92}

Background Advances in stroke care like most innovations in medicine have happened gradually, over time, in increments and have typically benefitted from the collaborative efforts of a diverse group with a common goal. The CLOT summit inaugurated in 2015 created an ecosystem guided by these principles to evaluate and develop pathways for enhancing stroke care.

Methodology Three core groups are invited to participate. These include scientists involved in clot research, engineers involved in device development and physicians involved in interventional and medical treatment of acute ischemic stroke. The initial format followed a focused group discussion and presentation on key topics leading to development of targeted projects. In subsequent years the outcome from previous year's summit was evaluated and a future course in basic and clinical research charted. A key clinical component was improving procedural performance and defining unmet needs.

Results To date, the output has covered several areas. Basic clot research: ex vivo clot analysis resulted in five registries in the US (one) and the EU (four) with the aim of multicenter collection of thrombectomy specimen with centralized analysis. This has yielded several publications in the JNIS and elsewhere. Procedure optimization: Pre-intervention imaging analysis involved multiple contributions on thrombus imaging using CT/CTA/MR. In-Vitro modeling for thrombectomy procedures centered around procedural aspects (tortuosity, the effects of flow and pressure, embolization to new territory) and clot interaction (fibrinous or calcified clots versus friable clots). Progress in device iteration was part of procedure optimization based on in-vitro modeling and review of challenging cases highlighting a potential unmet solution. Clinical Studies: These were a product of the first two and involve clinical studies related to new devices and clinical registry for calcified clots.
Conclusion The CLOT summit represents a novel incubator platform bringing together expertise in a coordinated fashion that has resulted in meaningful basic and clinical contribution to the evolving stroke care. Key outcomes of the summit over the first 5 -years are presented.

Disclosures A. Rai: 2; C; Cerenovus, Microvention, Stryker Neurovascular. J. Fiehler: 2; C; Cerenovus. R. McCarthy: None. A. Siddiqui: 2; C; Cerenovus. D. Liebeskind: 2; C; Cerenovus. W. Hacke: 2; C; Cerenovus.

\section{E-057 CLOT PERVIOUSNESS BUT NOT DENSITY IS ASSOCIATED WITH FIRST-PASS RECANALIZATION OF ASPIRATION THROMBECTOMY IN THE COMPASS TRIAL}

${ }^{1} \mathrm{M}$ Mokin*, ${ }^{2} \mathrm{M}$ Waqas, ${ }^{3} \mathrm{~J}$ Fifi, ${ }^{3} \mathrm{R}$ De Leacy, ${ }^{4} \mathrm{D}$ Fiorella, ${ }^{2} \mathrm{E}$ Gu, ${ }^{2} \mathrm{E}$ Levy, ${ }^{2} \mathrm{~K}$ Snyder, ${ }^{5} \mathrm{R}$ Hanel, ${ }^{5} \mathrm{~A}$ Aghaebrahim, ${ }^{6} \mathrm{~K}$ Woodward, ${ }^{6} \mathrm{H}$ Hixson, ${ }^{7} \mathrm{M}$ Chaudry, ${ }^{8} \mathrm{~A}$ Spiotta, ${ }^{9} \mathrm{~A}$ Rai, ${ }^{10} \mathrm{D}$ Frei, ${ }^{11} \mathrm{~J}$ Delgado Almandoz, ${ }^{12} \mathrm{M}$ Kelly, ${ }^{13} \mathrm{~A}$ Arthur, ${ }^{14} \mathrm{~B}$ Baxter, ${ }^{15} \mathrm{~J}$ English, ${ }^{16} \mathrm{I}$ Linfante, ${ }^{17} \mathrm{~K}$ Fargen, ${ }^{7} \mathrm{~A}$ Turk III, ${ }^{2} \mathrm{~A}$ Siddiqui, ${ }^{3} \mathrm{~J}$ Mocco. ${ }^{1}$ University of South Florida, Tampa, FL; ${ }^{2}$ University of Buffalo, Buffalo, NY; ${ }^{3}$ Icahn School of Medicine at Mount Sinai, New York, NY; ${ }^{4}$ Stony Brook University, Stony Brook, NY; ${ }^{5}$ Baptist Medical Center, Jacksonville, FL; ${ }^{6}$ Fort Sanders Regional Medical Center, Knoxville, TN; ${ }^{7}$ Greenville Health System, Greenville, SC; ${ }^{8}$ Medical University of South Carolina, Charleston, SC; ${ }^{9}$ West Virginia University, Morgantown, WV; ${ }^{10}$ Swedish Medical Cente, Englewood, CO; ${ }^{11}$ Abbott Northwestern Hospital, Minneapolis, MN; ${ }^{12}$ University of Saskatchewan, Saskatoon, SK, CANADA; ${ }^{13}$ University of South Florida, Memphis, TN; ${ }^{14}$ Erlanger Medical Center, Chatanooga, TN; ${ }^{15}$ California Pacific Medical Center, San Francisco, CA; ${ }^{16}$ Baptist Cardiac and Vascular Institute, Miami, FL; ${ }^{17}$ Wake Forest Baptist Medical Center, Winston-Salem, NC

\subsection{6/neurintsurg-2020-SNIS.93}

Background Imaging clot characteristics such as clot density and perviousness (the latter is defined as a difference in regional clot density values between computed tomography angiography (CTA) and non-contrast CT [NCCT]), can be used as an imaging marker characterizing red blood cell and fibrin composition of the clot serving. We aimed to examine whether clot density and perviousness were associated with angiographic outcomes of aspiration and stent retriever thrombectomy in the COMPASS Trial: a Direct Aspiration First Pass Technique trial.

Methods Clot density (Hounsfield units, HU) and perviousness were measured by two operators who were blind to all the final angiographic and clinical outcomes except for the knowledge of stroke laterality. NCCT and CTA images were co-registered to accurately localize clot on both imaging modalities. The values were then matched with angiographic and clinical outcome data of the first pass for each randomization arm. Univariate and multivariate analysis was carried out to assess the association of clot density and perviousness using SPSS version 25 .

Results Of the original 270 patients included in the COMPASS trial, 165 were eligible for the post-hoc analysis (81 patients in the aspiration first and 84 in the stent retriever first groups). There was no difference between the groups in regards to gender distribution, age, laterality and side of large vessel occlusion, smoking status of patients, and comorbidities. There was also no difference between the aspiration and stent retriever first randomization groups in regards to baseline clot Hounsfield units (HU) on NCCT (49.9 \pm 8.2 vs. $47.8 \pm 8.7, \mathrm{P}=0.11)$, and perviousness $(26.84 \pm 21.8$ vs. $22.8 \pm 19.9, \mathrm{P}=0.20)$. For the aspiration first group, there was a difference in mean perviousness values among patients who achieved TICI $2 \mathrm{c}-3$ vs. TICI $2 \mathrm{~b}$ vs. TICI $0-2 \mathrm{a}(33.1$ $\pm 26,35.9 \pm 25.1$, and $19.0 \pm 14.2$, respectively; $\mathrm{P}=0.016$ ). 
There was no difference between clot HU density on NCCT among these 3 groups $(48.8 \pm 8.5,50.1 \pm 7.2$, and 51.0 $\pm 8.4, \mathrm{P}=0.56$ ). In the stent retriever first group, there was no difference in perviousness or HU density of clot in patients with TICI $2 \mathrm{c} / 3$, TICI $2 \mathrm{~b}$ or TICI $0-2 \mathrm{a}$ after first pass (perviousness 21.6 $\pm 17.0,22.4 \pm 18.04$ and 22.6 $\pm 22.9, \mathrm{P}=0.97$; HU on NCCT $48.7 \pm 9.1,49.7 \pm 6.5$ and 46.7 $\pm 9.0, \mathrm{P}=0.47)$. In multivariate analysis using a model that included use of intravenous tPA, balloon guide catheter use, onset to groin puncture and age, perviousness of more than 10 was the only independent factor predictive of successful recanalization (defined as TICI 2b-3) after first pass in the aspiration first group with odds ratio of 3.4 (95\% CI $1.0-12.0)$. We did not find any significant predictors of successful reperfusion (TICI 2b-3) after first pass in the stent retriever first group.

Conclusions Clot perviousness values are associated with first pass angiographic success in patients treated with the aspiration first approach for thrombectomy. Additional research is needed to determine if clot perviousness may be used to identify patients who are more likely to have successful recanalization with aspiration when deciding between aspiration versus stent retriever first approaches.

Disclosures M. Mokin: 1; C; NIH R21NS109575. 2; C; Medtronic, Canon medical, Cerenovus. 4; C; Serenity medical, Synchron, VICIS, Endostream. M. Waqas: None. J. Fifi: 1; C; Stryker, Penumbra, Microvention. 4; C; Cerebrotech, The Stroke Project. R. De Leacy: 1; C; Penumbra. 6; C; Cerenovus, Siemens. D. Fiorella: 1; C; Penumbra, Cerenovus, Stryker. 6; C; Genentech, Shape Memory Medical. E. Gu: None. E. Levy: 6; C; Penumbra, NextGen Biologics, Rapid Medical, Cognition Medical, Three Rivers Medical, Stryker, MedX, Endostream Medical. K. Snyder: 6; C; Penumbra, Canon Medical Systems, Medtronic, Jacobs Institute; and other from Neurovascular Diagnostics. R. Hanel: 6; C; Penumbra, Endostream, Cerebrotech, Synchron, InNeuroCo, Medtronic, Microvention, Stryker, Cerenovus; Elum, Three Rivers. A. Aghaebrahim: None. K. Woodward: 6; C; Penumbra. H. Hixson: 6; C; Penumbra. M. Chaudry: 6; C; Penumbra, Pulsar Vascular, Medtronic, Microvention, Codman, Blockade. A. Spiotta: 6; C; Penumbra, Pulsar Vascular, Stryker, Microvention. A. Rai: 6; C; Penumbra, Microvention, Stryker. D. Frei: 6; C; Penumbra, Cerenovus, Stryker, Genentech, Shape Memory Medical, Siemens. J. Delgado Almandoz: 6; C; Penumbra, Medtronic. M. Kelly: 6; C; Penumbra, Medtronic, Endostream. A. Arthur: 6; C; Penumbra. B. Baxter: 6; C; Penumbra, Medtronic, Stryker, Viz, and 880 Medical. J. English: 6; C; Penumbra, Medtronic, Stryker, Route 92 Medical. I. Linfante: 6; C; Penumbra, Medtronic, Stryker, Microvention, InNeuroCo, andThree Rivers. K. Fargen: 6; C; Penumbra and Cerebrotech. A. Turk III: 6; C; Penumbra, Pulsar Vascular, Codman, Microvention, Medtronic, Blockade. A. Siddiqui: 6; C; Amnis Therapeutics, Serenity Medical, Silk Road Medical, Rebound Therapeutics, Penumbra Medtronic, Three Rivers Medical, Microvention, Imperative Care, Cerenovus, Endostream Medical, StimMed, Claret M. J. Mocco: 6; C; Penumbra, Cerebrotech, Rebound Therapeutics, TSP, Lazarus Effect, Medina, Pulsar Vascular, Blockade.

\section{E-058 \\ SAFETY AND OUTCOMES OF INTRA-ARTERIAL TISSUE PLASMINOGEN ACTIVATOR AS AN ADJUNCTIVE TECHNIQUE FOR DISTAL EMBOLIZATION - INSIGHTS FROM STAR}

${ }^{1}$ E Almallouhi*, ${ }^{2} S$ Al kasab, ${ }^{2} \mathrm{~A}$ Alawieh, ${ }^{3} \mathrm{M}$ Psychogios, ${ }^{4} \mathrm{~A}$ Arthur, ${ }^{5} \mathrm{~K}$ Kim, ${ }^{6} \mathrm{R}$ De Leacy, ${ }^{7} \mathrm{~A}$ Rai, ${ }^{8} \mathrm{~S}$ Keyrouz, ${ }^{9} \mathrm{~K}$ Fargen, ${ }^{10} \mathrm{~T}$ Dumont, ${ }^{11} \mathrm{P}$ Kan, ${ }^{12} \mathrm{R}$ Starke, ${ }^{2} \mathrm{~A}$ Spiotta. ${ }^{1}$ Medical University of South Carolina, Charleston, SC; ${ }^{2}$ Neurosurgery, Medical University of South Carolina, Charleston, SC; ${ }^{3}$ Neuroradiology, Universitätsspital Basel, Basel, SWITZERLAND; ${ }^{4}$ Neurosurgery, University of Tennessee Health Science Center, Memphis, TN; ${ }^{5}$ Chonnam Natl Univ Hosp, Kwangju, Korea, Republic of; ${ }^{6}$ Neurosurgery, Icahn School of Medicine at Mount Sinai, New York, NY; ${ }^{7}$ West Virginia University, Morgantown, $W W_{i}{ }^{8}$ Washington University in St Louis, St Louis, MO; ${ }^{9}$ Wake Forest University, Winston-Salem, NC: ${ }^{10}$ University of Arizona, Tucson, AZ; ${ }^{11}$ Baylor University, Houston, $T X ;{ }^{12}$ Neurosurgery, Medical University of South Carolina, Miami, FL

\subsection{6/neurintsurg-2020-SNIS.94}

Introduction Early studies have shown that using intra-arterial thrombolysis is effective in achieving recanalization in patients with acute ischemic stroke. However, the using intraarterial thrombolysis was associated with a high rate of symptomatic hemorrhagic transformation $(\mathrm{sICH})$. In current practice time, intra-arterial thrombolysis using tissue plasminogen activator (IA-tPA) can be used as an adjunctive technique in the setting of persistent distal occlusion during mechanical thrombectomy (MT).

Methods We used data from the Stroke Thrombectomy and Aneurysm Registry (STAR), which included the prospectively maintained databases from 28 thrombectomy-capable stroke centers in the US, Europe, and Asia. We included only consecutive patients who received MT using second generation thrombectomy devices. Then, we identified patients who received 5-10 mg of IA-tPA injected directly to the target vessel because of persistent distal occlusion during MT. We collected the baseline characteristics, procedural metrics, rate of sICH, and long-term functional outcomes. We estimated a Generalized Linear Model (GLM) with logit link to assess predictors of sICH, successful recanalization (Thrombolysis in Cerebral Infarction score $\geq 2$ b), and favorable 90-day functional outcomes (modified Rankin scale $0-2$ at 90-day).

Results A total of 154 patients received IA-tPA out of 1608 thrombectomy patients who were included in this analysis. Median age was 68 (IQR 57-76) years; 79 (51.3\%) were females; 97 (63\%) were white. There was no difference in stroke severity measured by the National Institute of Health stroke scale (NIHSS) (17 vs. $15, \mathrm{P}=0.079$ ) or Alberta Stroke Program Early CT (ASPECT) score (9 vs. 9, $\mathrm{P}=0.16$ ) between patients in the IA-tPA group compared to the nonIA-tPA group, respectively. In addition, no difference was noted in the prevalence of stroke risk factors (hypertension, diabetes, atrial fibrillation, prior stroke) between both groups. IA-tPA group had less patients who have received IV-tPA $(33.8 \%$ vs. $54.9 \%, \mathrm{P}<0.001)$ and longer onset-to-groin time (281 vs. $249 \mathrm{~min}, \mathrm{P}=0.003$ ) (table 1). Patients in the IA-tPA group achieved similar rates of successful recanalization (73.4\% vs. $74.6 \%, \mathrm{P}=0.736)$ and favorable functional outcomes $(40.9 \%$ vs. $40.8 \%, \mathrm{P}=0.976)$. On multivariate analysis, using IA-tPA was not independently associated with sICH (RR 0.761, 95\% CI 0.323-1.798, $\mathrm{P}=0.535$ ), successful recanalization (OR $0.888,95 \%$ CI $0.589-1.339, \mathrm{P}=0.572$ ), or favorable functional outcomes (OR 1.065, 95\% CI $0.72-$ $1.575, \mathrm{P}=0.752)$. 